Discrete Comput Geom 31:275-286 (2004)

DOI: $10.1007 / \mathrm{s} 00454-003-2879-8$

\title{
Expanding Polynomials and Connectedness of Self-Affine Tiles*
}

\author{
Ibrahim Kirat, ${ }^{1}$ Ka-Sing Lau, ${ }^{2}$ and Hui $\mathrm{Rao}^{3}$ \\ ${ }^{1}$ Department of Mathematics, Istanbul Technical University, \\ 34469 Maslak-Istanbul, Turkey \\ ibkst@yahoo.com \\ ${ }^{2}$ Department of Mathematics, The Chinese University of Hong Kong, \\ Shatin, Hong Kong \\ kslau@math.cuhk.edu.hk \\ ${ }^{3}$ Department of Mathematics, Wuhan University, \\ Wuhan, People's Republic of China \\ raohui69@public.wh.hb.cn
}

\begin{abstract}
Little is known about the connectedness of self-affine tiles in $\mathbb{R}^{n}$. In this note we consider this property on the self-affine tiles that are generated by consecutive collinear digit sets. By using an algebraic criterion, we call it the height reducing property, on expanding polynomials (i.e., all the roots have moduli $>1$ ), we show that all such tiles in $\mathbb{R}^{n}, n \leq 3$, are connected. The problem is still unsolved for higher dimensions. For this we make another investigation on this algebraic criterion. We improve a result of Garsia concerning the heights of expanding polynomials. The new result has its own interest from an algebraic point of view and also gives further insight to the connectedness problem.
\end{abstract}

\section{Introduction}

Let $M_{n}(\mathbb{Z})$ denote the set of $n \times n$ matrices with entries in $\mathbb{Z}$, and let $A \in M_{n}(\mathbb{Z})$ be expanding, that is, all eigenvalues of $A$ have moduli $>1$. Suppose $|\operatorname{det} A|=q$, we let $\mathcal{D}=\left\{0, d_{1}, \ldots, d_{q-1}\right\} \subseteq \mathbb{Z}^{n}$ be a set of $q$ distinct vectors, and call it a q-digit set. It is well known that there exists a unique compact set $T=T(A, \mathcal{D})$ satisfying the set-valued

\footnotetext{
* The first author was supported by a grant from the Wavelets Strategic Research Programme, National University of Singapore. The other two authors were partially supported by an HK RGC grant, a Direct Grant from the CUHK. The third author was partially supported by CNSF 19901025.
} 
equation

$$
A T=\bigcup_{d \in \mathcal{D}}(T+d) .
$$

The set $T$ can be expressed explicitly by $T=\left\{\sum_{i=1}^{\infty} A^{-i} x_{i}: x_{i} \in \mathcal{D}\right\} . T$ is called an integral self-affine tile if $T$ has a nonvoid interior. For such a tile there exists a subset $\mathcal{J} \subseteq \mathbb{Z}^{n}$, we call it a tiling set, such that

$$
T+\mathcal{J}=\mathbb{R}^{n} \quad \text { and } \quad(T+t)^{\circ} \cap\left(T+t^{\prime}\right)^{\circ}=\emptyset, \quad t \neq t^{\prime}, \quad t, t^{\prime} \in \mathcal{J} .
$$

Recently the geometric and algebraic properties of the self-affine tiles have been studied extensively in literature. However, knowledge on the connectedness of the tiles is still very limited. In [O] Odlyzko gave a characterization of a tile $T$ in $\mathbb{R}$ to be the finite union of intervals by using the strict product form of the digits. Bandt and Gelbrich [BG] investigated the disk-like tiles in $\mathbb{R}^{2}$ for $|\operatorname{det} A|=2$ or 3 and new progress has been obtained by Bandt and Wang in [BW]. In $\mathbb{R}^{n}$ the only known result was by Hacon et al. [H], which says that all two-digit tiles $T$ are pathwise connected (actually such tiles can be filled up by space filling curves).

In [KL] Kirat and Lau studied the particular class of integral self-affine tiles that are generated by the collinear digit sets of the form $\{0, v, 2 v, \ldots,(q-1) v\}, v \in \mathbb{Z}^{n} \backslash\{0\}$. They made use of the following algebraic property of the characteristic polynomial $f(x)$ of $A$ to determine the connectedness of $T(A, \mathcal{D})$. Let $\mathbb{Z}[x]$ denote the class of polynomials with integer coefficients. We say that a monic polynomial $f(x) \in \mathbb{Z}[x]$ with $|f(0)|=q$ has the height reducing (HR) property if there exists $g(x) \in \mathbb{Z}[x]$ such that

$$
g(x) f(x)=x^{k}+a_{k-1} x^{k-1}+\cdots+a_{1} x \pm q,
$$

with $\left|a_{i}\right| \leq q-1, i=1, \ldots, k-1$.

Theorem 1.1 [KL]. Let $A \in M_{n}(\mathbb{Z})$ be expanding with $|\operatorname{det} A|=q$ and let $\mathcal{D}=$ $\{0, v, 2 v, \ldots,(q-1) v\}$ be a collinear digit set in $\mathbb{Z}^{n}$. Suppose the characteristic polynomial $f(x)$ of $A$ has the HR-property, then $T$ is connected.

We call a polynomial $f(x) \in \mathbb{Z}[x]$ an expanding polynomial if all its roots have moduli greater than 1. It is easy to show that all monic, expanding integer polynomials of degree 2 have this property $[\mathrm{KL}]$. Hence, by Theorem 1.1 , all the tiles in $\mathbb{R}^{2}$ with collinear digits are connected. Also in $[\mathrm{KL}]$ it is shown explicitly that the expanding polynomials of degree 3 with constant terms equal to 2,3, 4, or 5 all have the HR-property. Motivated by that, we prove here (Theorem 2.7):

Theorem 1.2. All the expanding integer monic polynomials of degree 3 have the HRproperty. Consequently, if $T$ is a self-affine tile in $\mathbb{R}^{3}$ generated by an expanding integer matrix $A$ with $|\operatorname{det} A|=q$ and collinear digit set $\mathcal{D}=\{0, v, 2 v, \ldots,(q-1) v\}, v \in \mathbb{Z}^{3}$, then $T$ is connected.

The proof of the theorem is elementary, however, it cannot be extended to higher degrees directly. In Lemma 1.6 of [G2] Garsia proved a statement weaker than the 
HR-property: for an expanding polynomial $f(x) \in \mathbb{Z}[x]$ with $|f(0)|=q$, there exists $g(x) \in \mathbb{Z}[x]$ such that

$$
g(x) f(x)=a_{k} x^{k}+\cdots+a_{1} x \pm q, \quad \text { where } \quad\left|a_{j}\right| \leq q, \quad \forall j=1, \ldots, k .
$$

His proof makes use of the pigeonhole principle and is simple. Here we improve Garsia's result by employing a geometric argument of the tiling set:

Theorem 1.3. Let $f(x) \in \mathbb{Z}[x]$ be an irreducible expanding monic polynomial with $|f(0)|=q$. Then there exists $g(x) \in \mathbb{Z}[x]$ such that

$$
h(x)=f(x) g(x)=a_{k} x^{k}+a_{k-1} x^{k-1}+\cdots+a_{1} x \pm q,
$$

where $\left|a_{i}\right| \leq q-1$ for $i=1, \ldots, k$.

Still the result falls short of the HR-property on the leading coefficient. We therefore make the following conjecture:

Conjecture. All monic expanding polynomials $f(x) \in \mathbb{Z}[x]$ have the HR-property.

If this is true, then all the self-affine tiles generated by consecutive collinear digit sets are connected.

\section{Expanding Polynomials of Degree 3}

Let $f(x)$ and $\tilde{f}(x)$ denote the characteristic polynomials of $A$ and $-A \in M_{n}(\mathbb{Z})$, respectively. It is easy to see that $\tilde{f}(x)=(-1)^{n} f(-x)$. This enables us to restrict our attention to the degree 3 characteristic polynomials with positive constant terms, i.e., $\operatorname{det} A<0$ (see Lemma 5.6 of [KL] and the remark before the lemma). Hence in the following we only consider

$$
f(x)=x^{3} \pm a x^{2} \pm b x+q,
$$

where $a, b \geq 0, a b \neq 0$, and $q \geq 2$ (the case $a b=0$ is trivial). We want to determine the coefficients $a, b$ so that $f$ is an expanding polynomial. We begin with two elementary lemmas.

Lemma 2.1. Suppose $f(x)$ is of the form (2.1) and is expanding, then the following holds:

$$
f(1), f(-1), f(q)>0 \text { and } f(-q)<0 .
$$

Proof. Note that $f(0)=q>0$. If $f(1)$ or $f(-1) \leq 0$, then the intermediate value theorem will imply that there is a root in $[-1,1]$. This will contradict the expanding property.

If $f(q) \leq 0$ or $f(-q) \geq 0$, then there will be a root $\theta$ with $|\theta| \geq q$. Since the product of the three roots is $q$, one of the other two roots must have modulus $\leq 1$. This is impossible. 
Lemma 2.2. Let $f(x)$ be of the form (2.1). Suppose $f(x)$ has a real root in $(-q,-1)$ or $(1, q)$ and has no real root in $[-1,1]$. Then $f(x)$ is expanding.

Proof. Let $\theta_{i}, i=1,2,3$, be the roots with $\theta_{1}$ real and $1<\left|\theta_{1}\right|<q$. If $\theta_{2}, \theta_{3}$ are real, then by assumption they have moduli $>1$ and the result is trivial. Otherwise $\theta_{2}, \theta_{3}$ are complex conjugates and $q=\theta_{1} \theta_{2} \theta_{3}=\theta_{1}\left|\theta_{2}\right|^{2}$. This implies that $\left|\theta_{2}\right|=\left|\theta_{3}\right|>1$ and $f$ is expanding.

We now divide the classification of the degree 3 expanding polynomials into four different cases.

Proposition 2.3. Let $f(x)=x^{3}-a x^{2}+b x+q$ as in (2.1). Then $f(x)$ is expanding if and only if $q \geq a+b+2$.

Proof. For the necessity, we use Lemma 2.2 to show that

$$
0<f(-1)=-(1+a+b)+q .
$$

For the sufficiency, we see that $q \geq a+b+2$ implies that $f(-q)<0, f(-1)>0$, hence there is a root in $(-q,-1)$. We next claim that $f(x)>0$ for $x \in[-1,1]$. Indeed, it is clear for $x \in[0,1]$. For $x \in[-1,0]$, we have

$$
\begin{aligned}
f(x) & =x^{3}-a x^{2}+b x+q \\
& \geq x^{3}-a x^{2}+b x+a+b+2 \\
& =x^{3}+a\left(1-x^{2}\right)+b(1+x)+2>0 .
\end{aligned}
$$

Lemma 2.2 implies that $f$ is expanding.

Proposition 2.4. Let $f(x)=x^{3}-a x^{2}-b x+q$ as in (2.1). Then $f(x)$ is expanding if and only if $q \geq a+b$.

Proof. The proof is similar to the above. For the necessity, we use $0<f(1)=$ $1-a-b+q$. For the sufficiency, we observe that $f(-q)<0, f(-1)>0$, hence there exists a root in $(-q,-1)$. Also there is no root in $[-1,1]$ : this is because $f(x)>0$ trivially for $x \in[-1,0]$, and for $x \in[0,1]$,

$$
\begin{aligned}
f(x) & \geq x^{3}-a x^{2}-b x+a+b \\
& =x^{3}+a\left(1-x^{2}\right)+b(1-x) \geq 0 .
\end{aligned}
$$

Hence $f$ is expanding by Lemma 2.2 .

Proposition 2.5. Let $f(x)=x^{3}+a x^{2}-b x+q$ as in (2.1). Then $f(x)$ is expanding if and only if

(i) $a=q-1, b \leq q-2$ or

(ii) $a<q-1, b \leq q+a$. 
Proof. For the necessity, we use $f(-q)<0$ to see that $a \leq q-1$. Case (i) follows immediately; for case (ii) we make use of $f(1)>0$. To prove the sufficiency, we observe that either one of the conditions implies that $f(-q)<0, f(-1)>0$, hence there is a root in $(-q,-1)$. For $x \in(-1,0]$ it is clear that $f(x)>0$ and for $x \in(0,1)$,

$$
\begin{aligned}
f(x) & \geq x^{3}+a x^{2}-(a+q) x+q \\
& =x^{3}+(1-x)(q-a x)>0 .
\end{aligned}
$$

Hence $f(x)$ is expanding by Lemma 2.2 .

Proposition 2.6. Let $f(x)=x^{3}+a x^{2}+b x+q$ as in (2.1). Then $f(x)$ is expanding if and only if

(i) $a=q+1, q+2 \leq b \leq 2 q-1$; or

(ii) $a=q, 2 \leq b \leq 2 q-2$; or

(iii) $a \leq q-1, b \leq q+a-2$.

Proof. For the necessity, we use $f(-1)>0$ and $f(-q)<0$ to show that $a \leq q+1$. We then make use of $f(-q)<0$ again to imply cases (i) and (ii); case (iii) follows from $f(-1)>0$. To prove the reverse implication, we observe that any one of cases (i)-(iii) will imply $f(-q)<0, f(-1)>0$, hence there is a root in $(-q,-1)$. Also $f(x)>0$ on $(0,1]$ and for $x \in[-1,0]$,

$$
\begin{aligned}
f(x) & \geq x^{3}+a x^{2}+(a+q-2) x+q \\
& =x^{3}+2 x^{2}+(1+x)(q+(a-2) x)>0 .
\end{aligned}
$$

This implies $f(x)$ is expanding by Lemma 2.2.

We conclude this section with the following main result:

Theorem 2.7. Let $f(x) \in \mathbb{Z}[x]$ be a degree 3 , monic expanding polynomial. Then $f(x)$ has the HR-property.

Proof. We assume that $f(x)$ is as in (2.1). For the $f(x)$ that $a$ or $b>q-1$, as in Propositions 2.5(ii) and 2.6(i)-(iii), we need to find a $g(x) \in \mathbb{Z}[x]$ to reduce the coefficients to have moduli $\leq q-1$. We assume that $q \geq 4$ (because of case II in the following); for the case $q=2,3$, the theorem is true by Table 1 in the Appendix.

(I) In Proposition 2.5(ii), $a<q-1$ and we divide the $b \leq q+a$ into two cases. If $b=q+a$, we let $g(x)=\left(x^{2}+1\right)(x+1)$, then

$$
g(x) f(x)=x^{6}+(a+1) x^{5}+(1-q) x^{4}+x^{3}-a x+q .
$$

If $q \leq b<q+a$, we let $g(x)=x+1$, then

$$
g(x) f(x)=x^{4}+(a+1) x^{3}+(a-b) x^{2}+(q-b) x+q .
$$


(II) In Proposition 2.6(i), $a=q+1$ and we divide the $b$ into three cases. If $b=(q+2)$, we let $g(x)=(x-1)^{2}$, then

$$
g(x) f(x)=x^{5}+(q-1) x^{4}-(q-1) x^{3}-3 x^{2}-(q-2) x+q .
$$

If $b=2 q-1$, we let $g(x)=\left(x^{2}+1\right)(x-1)^{2}$, then

$$
g(x) f(x)=x^{7}+(q-1) x^{6}-x^{5}-(q-2) x^{4}-3 x^{3}-(q-3) x^{2}-x+q .
$$

If $q+2<b<2 q-1$, we take $g(x)=(x-1)^{2}\left(x^{2}+1\right)\left(x^{3}+1\right)$, then

$$
\begin{aligned}
g(x) f(x)= & x^{10}+(q-1) x^{9}+(b-2 q) x^{8}+(3 q-2 b+1) x^{7}+(2 b-3 q-2) x^{6} \\
& +(q-b+1) x^{5}+(q-b) x^{4}+(2 b-3 q-1) x^{3} \\
& +(3 q-2 b+1) x^{2}+(b-2 q) x+q,
\end{aligned}
$$

and the HR-property is satisfied.

(III) For Proposition 2.6(ii) and (iii), we take $g(x)=x-1$, then

$$
g(x) f(x)=x^{4}+(a-1) x^{3}+(b-a) x^{2}+(q-b) x+q .
$$

These take care of all the cases and the theorem is proved.

It follows immediately from this and Theorem 1.1 that any self-affine tile in $\mathbb{R}^{3}$ generated by an expanding integer matrix $A$ with $|\operatorname{det} A|=q$ and collinear digit set $\mathcal{D}=\{0, v, \ldots,(q-1) v\}$ is connected. As an illustration of Theorem 2.7, we attach in the Appendix the degree 3 expanding integer polynomial for $q=2,3,4,5$ with $a$ or $b>q-1$ and the corresponding polynomials after the height is reduced. Note that the table for $q=5$ includes all the cases in the theorem and the factors $g$ we used in the proof.

\section{Another Reduction of the Coefficients}

In this section we need some notions on the tiles introduced by Lagarias and Wang in $[\mathrm{LW} 2]$. Let $\mathbb{Z}[A, \mathcal{D}]$ be the integer lattice generated by $\mathcal{D}, \ldots, A^{n-1} \mathcal{D}$. If $\mathbb{Z}[A, \mathcal{D}]=\mathbb{Z}^{n}$, we say that $(A, \mathcal{D})$ is a primitive pair (or $\mathcal{D}$ is primitive in short) and the tile $T(A, \mathcal{D})$ is a primitive tile. The following proposition was proved in [LW2, Corollary 6.2].

Proposition 3.1. Suppose that the expanding matrix $A \in M_{n}(\mathbb{Z})$ is irreducible. Then for any primitive digit set $\mathcal{D}$ which is a complete set of coset representatives of $\mathbb{Z}^{n} / A \mathbb{Z}^{n}$, the tile $T(A, \mathcal{D})$ tiles $\mathbb{R}^{n}$ with $\mathbb{Z}^{n}$, i.e., $\mathcal{J}=\mathbb{Z}^{n}$ is a tiling set.

If the tile $T(A, \mathcal{D})$ is not primitive, then we can easily adjust it by the following proposition (Lemma 2.1 of [LW2]).

Proposition 3.2. Let $T(A, \mathcal{D})$ be an integral self-affine tile in $\mathbb{R}^{n}$ with $\mathbb{Z}[A, \mathcal{D}]=B \mathbb{Z}^{n}$. Define

$$
\widetilde{A}=B^{-1} A B \in M_{n}(\mathbb{Z}), \quad \widetilde{\mathcal{D}}=B^{-1} \mathcal{D} \in \mathbb{Z}^{n},
$$

then $T(\widetilde{A}, \widetilde{\mathcal{D}})$ is a primitive tile and satisfies $T(\widetilde{A}, \widetilde{\mathcal{D}})=B^{-1} T(A, \mathcal{D})$. 
Now let $f(x)=x^{n}+c_{n-1} x^{n-1}+\cdots+c_{1} x+q \in \mathbb{Z}[x]$ be an expanding polynomial, we consider the corresponding companion matrix

$$
A=\left[\begin{array}{ccccc}
0 & 1 & 0 & & \\
& \ddots & \ddots & \ddots & \\
& & 0 & 1 & 0 \\
& & & 0 & 1 \\
-q & \cdots & \cdots & \cdots & -c_{n-1}
\end{array}\right] .
$$

Lemma 3.3. Let $f(x) \in \mathbb{Z}[x]$ be an expanding monic polynomial, then there exists an irreducible $A \in M_{n}(\mathbb{Z})$ and $\mathcal{D}=\{0, v, \ldots,(q-1) v\} \subset \mathbb{Z}^{n}$ such that

(i) A has $f(x)$ as its characteristic polynomial;

(ii) $\mathcal{D}$ is a complete set of coset representatives of $\mathbb{Z}^{n} / A \mathbb{Z}^{n}$.

Furthermore we can choose the above pair $(A, \mathcal{D})$ such that the tiling set $\mathcal{J}=\mathbb{Z}^{n}$.

Proof. Let $A$ be defined as in (3.1) and let $A^{*}$ be the adjoint matrix of $A$ (i.e., $A A^{*}=q I$ ), then

$$
A^{*}=\left[\begin{array}{ccccc}
-c_{1} & & \cdots & -c_{n-1} & -1 \\
q & & & & \\
& q & & \mathbf{0} & \vdots \\
\mathbf{0} & & \ddots & & \\
& & & q & 0
\end{array}\right] .
$$

Let $v=[0, \ldots, 0,1]^{t}$ and let $\mathcal{D}=\{0, v, \ldots,(q-1) v\}$. Notice that $\left|\mathbb{Z}^{n} / A \mathbb{Z}^{n}\right|=$ $|\operatorname{det} A|=q$. Suppose $\mathcal{D}$ is not a complete coset representative of $\mathbb{Z}^{n} / A \mathbb{Z}^{n}$, then there exists $1 \leq \ell \leq q-1$ such that $\ell v=A w$. Hence

$$
[-\ell, 0, \ldots, 0]^{t}=\ell A^{*} v=A^{*} A w=q w .
$$

This implies that $q$ divides $\ell$ and is a contradiction. Hence $\mathcal{D}$ is complete in $\mathbb{Z}^{n} / A \mathbb{Z}^{n}$.

For the second part we see that $A$ is irreducible. If the pair $(A, \mathcal{D})$ is primitive, then we are done. Otherwise, we can find $B, \widetilde{\widetilde{A}}, \underset{\widetilde{D}}{\widetilde{\mathcal{D}}}$ as in Proposition 3.2 such that $(\widetilde{A}, \widetilde{\mathcal{D}})$ is a primitive pair. We need to see that $(\widetilde{A}, \widetilde{\mathcal{D}})$ will satisfy (i) and (ii).

Indeed, $\widetilde{A}=B^{-1} A B$ implies that $\widetilde{A}$ has $f(x)$ as its charateristic polynomial. Let $\widetilde{v}=B^{-1} v$, then $\widetilde{\mathcal{D}}=\{0, \widetilde{v}, \ldots,(q-1) \widetilde{v}\}$. It remains to prove that $\widetilde{\mathcal{D}}$ is complete in $\mathbb{Z}^{n} / \widetilde{A} \mathbb{Z}^{n}$. Suppose this is not true, then there is an integer $1 \leq \ell \leq q-1$ such that $\ell \widetilde{v}=\widetilde{A} u$ for some $u \in \mathbb{Z}^{n}$. This implies

$$
\ell v=\ell B \tilde{v}=B \tilde{A} u=B B^{-1} A B u=A(B u)
$$

and contradicts that $\mathcal{D}$ is complete in $\mathbb{Z}^{n} / A \mathbb{Z}^{n}$ as proved in the previous paragragh.

The last statement follows from Proposition 3.1.

Let $\mathcal{D}_{0}=\{0\}$ and

$$
\mathcal{D}_{k}=\left\{\sum_{i=0}^{k-1} A^{i} x_{i}: x_{i} \in \mathcal{D}\right\}=\bigcup_{j=0}^{q-1}\left(j A^{k-1} v+\mathcal{D}_{k-1}\right), \quad k \geq 1 .
$$


Then $\mathcal{D}_{k} \subset \mathbb{Z}^{n}$ and $\mathcal{D}_{k}$ is increasing on $k$. Moreover, we have

Lemma 3.4. If $T(A, \mathcal{D})$ tiles $\mathbb{R}^{n}$ with tiling set $\mathcal{J}=\mathbb{Z}^{n}$, then $\left(\mathcal{D}_{k}+T\right)^{\circ} \cap \mathbb{Z}^{n} \subset \mathcal{D}_{k}$.

Proof. Let $u \in \mathbb{Z}^{n}$, then for any $k$, the tiling property of $\mathbb{Z}^{n}$ implies that either

$$
(u+T)^{\circ} \cap\left(\mathcal{D}_{k}+T\right)^{\circ}=\emptyset \quad \text { or } \quad(u+T)^{\circ} \subseteq\left(\mathcal{D}_{k}+T\right)^{\circ} .
$$

In the latter case, $u$ must be in $\mathcal{D}_{k}$ by the tiling property of $\mathbb{Z}^{n}$ again. Hence $u \in$ $\left(\mathcal{D}_{k}+T\right)^{\circ} \cap \mathbb{Z}^{n}$ implies that $u \in \mathcal{D}_{k}$ and the lemma follows.

Theorem 3.5. Let $f(x) \in \mathbb{Z}[x]$ be an irreducible, expanding monic polynomial with $|f(0)|=q$. Then there exists $g(x) \in \mathbb{Z}[x]$ such that

$$
h(x)=g(x) f(x)=a_{k} x^{k}+a_{k-1} x^{k-1}+\cdots+a_{1} x \pm q,
$$

where $\left|a_{i}\right| \leq q-1$ for $i=1, \ldots, k$.

Proof. Let $A$ and $\mathcal{D}=\{0, v, \ldots,(q-1) v\}$ be as in Lemma 3.3, then $\mathcal{J}=\mathbb{Z}^{n}$ is a tiling set for $T$ tiles (by Proposition 3.1). Since $A^{k} T=\mathcal{D}_{k}+T$ contains an arbitrarily large ball when $k$ tends to infinity and $\mathbb{Z}^{n}$ tiles $\mathbb{R}^{n}$, it follows from Lemma 3.4 that for $k$ large, $\mathcal{D}_{k}$ contains an arbitrarily long sequence of vectors of the form $\{x, x+v, \ldots, x+t v\}, x \in$ $\mathbb{Z}^{n}, t \in \mathbb{N}$. Assume that $m+1$ is the smallest integer such that $\mathcal{D}_{m+1}$ contains $q+1$ vectors $\{x, x+v, \ldots, x+q v\}$ for some $x \in \mathbb{Z}^{n}$. Note that $\mathcal{D}_{m+1}=\bigcup_{i=0}^{q-1}\left(i A^{m} v+\mathcal{D}_{m}\right)$, we claim that there exists $j, 0 \leq j \leq q-1$, and $i_{1} \neq i_{2}, 0 \leq i_{1}, i_{2} \leq q-1$, such that

$$
x+j v \in i_{1} A^{m} v+\mathcal{D}_{m}, \quad x+(j+1) v \in i_{2} A^{m} v+\mathcal{D}_{m} .
$$

If otherwise, there exists $i^{*}$ such that $x+j v \in i^{*} A^{m} v+\mathcal{D}_{m}$ for all $j=0,1, \ldots, q$. Let $x^{\prime}=x-i^{*} A^{m} v$, then $\mathcal{D}_{m}$ contains the sequence $\left\{x^{\prime}, x^{\prime}+v, \ldots, x^{\prime}+q v\right\}$, which contradicts the minimal assumption of $m$. Hence the claim is true.

We write

$$
\begin{aligned}
x+j v & =i_{1} A^{m} v+\sum_{i=0}^{m-1} r_{i} A^{i} v, & & 0 \leq r_{i} \leq q-1, \\
x+(j+1) v & =i_{2} A^{m} v+\sum_{i=0}^{m-1} s_{i} A^{i} v, & & 0 \leq s_{i} \leq q-1 .
\end{aligned}
$$

By subtracting the two expressions and rearranging, we have

$$
\left(i_{2}-i_{1}\right) A^{m} v+\sum_{i=1}^{m-1}\left(s_{i}-r_{i}\right) A^{i} v+\left(s_{0}-r_{0}-1\right) v=0 .
$$

Let

$$
h(x)=\sum_{i=0}^{m} a_{i} x^{i}:=\left(i_{2}-i_{1}\right) x^{m}+\sum_{i=1}^{m-1}\left(s_{i}-r_{i}\right) x^{i}+\left(s_{0}-r_{0}-1\right) .
$$


Then $\left|a_{0}\right| \leq q$ and $0 \leq\left|a_{i}\right| \leq q-1$ for $i=1, \ldots, m$. Since $f(x)$ is the characteristic polynomial of $A, f(A)=0$ by Cayley's theorem. Therefore we can reduce $h(A)$ to the form

$$
h(A)=\sum_{i=0}^{n-1} c_{i} A^{i}
$$

By (3.2)

$$
0=h(A) v=\sum_{i=0}^{n-1} c_{i} A^{i} v
$$

Since $T$ is a tile, $v, A v, \ldots, A^{n-1} v$ are independent, (3.4) implies that $c_{i}=0$, and by (3.3), $h(A)=0$. Since the characteristic polynomial $f$ is irreducible by assumption, $f$ must divide $h$, and the constant term of $h$ must be $\pm q$. The theorem follows by taking $g(x)=h(x) / f(x)$.

\section{Remarks}

It follows directly from the theorem that if $q=2$, then $f(x)$ has the HR-property, and hence a two-digit tile is connected. Of course, the second fact is known in $[\mathrm{H}]$. We also remark that for a give expanding integral matrix $A$ with $|\operatorname{det} A|=q$, the collinear digit sets $\{0, v, \ldots,(q-1) v\}$ may not give a tile (e.g., if $A=2 I$ is a $2 \times 2$ matrix, then for any collinear digit set $D, T$ is one-dimensional and cannot be a tile, however it is connected). Some sufficient conditions have been studied in Theorems 3.1 and 3.3 of [KL].

It is well known that in the one-dimensional case, if $A=[p]$ where $p$ is a prime, then $\mathcal{D}=\left\{0, d_{1}, \ldots, d_{p-1}\right\}$ is connected if and only if $\mathcal{D}=\{0, d, \ldots, d(p-1)\}$ for some $d \in \mathbb{Z}$. This is not true in higher dimensions. In fact Tan [T] has an interesting example that if $A$ is an expanding integer matrix with characteristic polynomial $x^{2}-x-3(|\operatorname{det} A|=$ 3) and if $\mathcal{D}=\left\{0, d_{1} v, d_{2} v\right\}$ with $v=[0,1]^{t}$ and $d_{1}<d_{2}$, then $T$ is connected if $\frac{8}{5} d_{1} \leq d_{2} \leq \frac{8}{3} d_{1}$; it is disconnected if $d_{2}<((\sqrt{13}-1) / 2) d_{1}$ or $d_{2}>((\sqrt{13}+5) / 2) d_{1}$.

It is known that an integer polynomial $f(x)=x^{2}+a x+q$ is exanding if and only if $|a| \leq q$ if $q \geq 2$, and $|a| \leq|q|-2$ if $q<0$ [BG], [KL]. It is not difficult to show that $[\mathrm{T}]$

$$
f(x) \text { has the HR-property if and only if }|a| \leq q \text { if } q \geq 1 \text {, and }|a| \leq|q|-1 \text { if } q<0 \text {. }
$$

In particular, $f(x)=x^{2}-x+1$ has the HR-property (multiply by $x+1$ ), but it is not expanding as the two roots are $(1 \pm \sqrt{-3}) / 2$.

Since the completion of the paper, Leung [L] has drawn our attention to the SchurCohn criterion [G1] for expanding polynomials. By using that, he found the explicit expression of the coefficients of the expanding polynomials (as in Proposition 2.6) up to degree 4 . Furthermore, by using a case by case test similar to Theorem 2.7, Akiyama and Gjini $[\mathrm{AG}]$ proved that the HR-property holds up to degree 4 . However, the problem for arbitrary degree remains unsolved. 


\section{Acknowledgements}

The authors thank the referee for many valuable suggestions. They thank Dr. B. Tan and Mr. K.S. Leung for many stimulating discussions. They are also grateful to Professors S. Akiyama and N. Gjini of informing them of their interesting work.

\section{Appendix}

The following tables list the degree 3 expanding integer polynomials with constant terms $q=2,3,4,5$ and some of the other terms have coefficients $>q-1$. We need to multiply factors $g(x)$ to reduce the heights as in Theorem 1.3. Note that all the factors $g(x)$ used are from the roots of unity.

Table 1. $f(0)=2,3$.

\begin{tabular}{lcc}
\hline \multicolumn{1}{c}{ c.p. $=f(x)$} & $g(x)$ & $h(x)=g(x) f(x)$ \\
\hline$x^{3}-2 x+2$ & $\left(x^{2}+1\right)(x+1)$ & $x^{6}+x^{5}-x^{4}+x^{3}+2$ \\
$x^{3}+2 x^{2}+2 x+2$ & $x-1$ & $x^{4}+x^{3}-2$ \\
$x^{3}-3 x+3$ & $\left(x^{2}+1\right)(x+1)$ & $x^{6}+x^{5}-2 x^{4}+x^{3}+3$ \\
$x^{3}+x^{2}-3 x+3$ & $x+1$ & $x^{4}+2 x^{3}-2 x^{2}+3$ \\
$x^{3}+x^{2}-4 x+3$ & $\left(x^{2}+1\right)(x+1)$ & $x^{6}+2 x^{5}-2 x^{4}+x^{3}-x+3$ \\
$x^{3}+2 x^{2}+3 x+3$ & $x-1$ & $x^{4}+x^{3}+x^{2}-3$ \\
$x^{3}+3 x^{2}+2 x+3$ & $x-1$ & $x^{4}+2 x^{3}-x^{2}+x-3$ \\
$x^{3}+3 x^{2}+3 x+3$ & $x-1$ & $x^{4}+2 x^{3}-3$ \\
$x^{3}+3 x^{2}+4 x+3$ & $x-1$ & $x^{4}+2 x^{3}+x^{2}-x-3$ \\
$x^{3}+4 x^{2}+5 x+3$ & $\left(x^{2}-x+1\right)(x-1)$ & $x^{6}+2 x^{5}-x^{4}+x-3$ \\
\hline
\end{tabular}

Table 2. $f(0)=4$.

\begin{tabular}{lcc}
\hline \multicolumn{1}{c}{ c.p. $=f(x)$} & $g(x)$ & $h(x)=g(x) f(x)$ \\
\hline$x^{3}-4 x+4$ & $\left(x^{2}+1\right)(x+1)$ & $x^{6}+x^{5}-3 x^{4}+x^{3}+4$ \\
$x^{3}+x^{2}-4 x+4$ & $x+1$ & $x^{4}+2 x^{3}-3 x^{2}+4$ \\
$x^{3}+x^{2}-5 x+4$ & $\left(x^{2}+1\right)(x+1)$ & $x^{6}+2 x^{5}-3 x^{4}+x^{3}-x+4$ \\
$x^{3}+2 x^{2}-4 x+4$ & $x+1$ & $x^{4}+3 x^{3}-2 x^{2}+4$ \\
$x^{3}+2 x^{2}-5 x+4$ & $x+1$ & $x^{4}+3 x^{3}-3 x^{2}-x+4$ \\
$x^{3}+2 x^{2}-6 x+4$ & $\left(x^{2}+1\right)(x+1)$ & $x^{6}+3 x^{5}-3 x^{4}+x^{3}-2 x+4$ \\
$x^{3}+2 x^{2}+4 x+4$ & $x-1$ & $x^{4}+x^{3}+2 x^{2}-4$ \\
$x^{3}+3 x^{2}+4 x+4$ & $x-1$ & $x^{4}+2 x^{3}+x^{2}-4$ \\
$x^{3}+3 x^{2}+5 x+4$ & $x-1$ & $x^{4}+2 x^{3}+2 x^{2}-x-4$ \\
$x^{3}+4 x^{2}+2 x+4$ & $x-1$ & $x^{4}+3 x^{3}-2 x^{2}+2 x-4$ \\
$x^{3}+4 x^{2}+3 x+4$ & $x-1$ & $x^{4}+3 x^{3}-x^{2}+x-4$ \\
$x^{3}+4 x^{2}+4 x+4$ & $x-1$ & $x^{4}+3 x^{3}-4$ \\
$x^{3}+4 x^{2}+5 x+4$ & $x-1$ & $x^{4}+3 x^{3}+x^{2}-x-4$ \\
$x^{3}+4 x^{2}+6 x+4$ & $x-1$ & $x^{4}+3 x^{3}+2 x^{2}-2 x-4$ \\
$x^{3}+5 x^{2}+6 x+4$ & $(x-1)^{2}$ & $x^{5}+3 x^{4}-3 x^{3}-3 x^{2}-2 x+4$ \\
$x^{3}+5 x^{2}+7 x+4$ & $\left(x^{2}+1\right)(x-1)^{2}$ & $x^{7}+3 x^{6}-x^{5}-2 x^{4}-3 x^{3}-x^{2}-x+4$ \\
\hline
\end{tabular}


Table 3. $f(0)=5$.

\begin{tabular}{lcc}
\hline \multicolumn{1}{c}{ c.p. $=f(x)$} & $g(x)$ & $h(x)=g(x) f(x)$ \\
\hline$x^{3}-5 x+5$ & $\left(x^{2}+1\right)(x+1)$ & $x^{6}+x^{5}-4 x^{4}+x^{3}+5$ \\
$x^{3}+x^{2}-5 x+5$ & $x+1$ & $x^{4}+2 x^{3}-4 x^{2}+5$ \\
$x^{3}+x^{2}-6 x+5$ & $\left(x^{2}+1\right)(x+1)$ & $x^{6}+2 x^{5}-4 x^{4}+x^{3}-x+5$ \\
$x^{3}+2 x^{2}+5 x+5$ & $x-1$ & $x^{4}+x^{3}+3 x^{2}-5$ \\
$x^{3}+2 x^{2}-5 x+5$ & $x+1$ & $x^{4}+3 x^{3}-3 x^{2}+5$ \\
$x^{3}+2 x^{2}-6 x+5$ & $x+1$ & $x^{4}+3 x^{3}-4 x^{2}-x+5$ \\
$x^{3}+2 x^{2}-7 x+5$ & $\left(x^{2}+1\right)(x+1)$ & $x^{6}+3 x^{5}-4 x^{4}+x^{3}-2 x+5$ \\
$x^{3}+3 x^{2}+5 x+5$ & $x-1$ & $x^{4}+2 x^{3}+2 x^{2}-5$ \\
$x^{3}+3 x^{2}-5 x+5$ & $x+1$ & $x^{4}+4 x^{3}-2 x^{2}+5$ \\
$x^{3}+3 x^{2}+6 x+5$ & $x-1$ & $x^{4}+4 x^{3}+3 x^{2}-x-5$ \\
$x^{3}+3 x^{2}-6 x+5$ & $x+1$ & $x^{4}+4 x^{3}-4 x^{2}-2 x+5$ \\
$x^{3}+3 x^{2}-7 x+5$ & $x+1$ & $x^{6}+4 x^{5}-4 x^{4}+x^{3}-3 x+5$ \\
$x^{3}+3 x^{2}-8 x+5$ & $\left(x^{2}+1\right)(x+1)$ & $x^{4}+3 x^{3}+x^{2}-5$ \\
$x^{3}+4 x^{2}+5 x+5$ & $x-1$ & $x^{4}+3 x^{3}+2 x^{2}-x-5$ \\
$x^{3}+4 x^{2}+6 x+5$ & $x-1$ & $x^{4}+3 x^{3}+3 x^{2}-2 x-5$ \\
$x^{3}+4 x^{2}+7 x+5$ & $x-1$ & $x^{4}+4 x^{3}-3 x^{2}+3 x-5$ \\
$x^{3}+5 x^{2}+2 x+5$ & $x-1$ & $x^{4}+4 x^{3}-2 x^{2}+2 x-5$ \\
$x^{3}+5 x^{2}+3 x+5$ & $x-1$ & $x^{4}+4 x^{3}-x^{2}+x-5$ \\
$x^{3}+5 x^{2}+4 x+5$ & $x-1$ & $x^{4}+4 x^{3}-5$ \\
$x^{3}+5 x^{2}+5 x+5$ & $x-1$ & $x^{4}+4 x^{3}+x^{2}-x-5$ \\
$x^{3}+5 x^{2}+6 x+5$ & $x-1$ & $x^{4}+4 x^{3}+2 x^{2}-2 x-5$ \\
$x^{3}+5 x^{2}+7 x+5$ & $x-1$ & $x^{4}+4 x^{3}+3 x^{2}-3 x-5$ \\
$x^{3}+5 x^{2}+8 x+5$ & $x-1$ & $x^{5}+4 x^{4}-4 x^{3}-3 x^{2}-3 x+5$ \\
$x^{3}+6 x^{2}+7 x+5$ & $(x-1)^{2}$ & $x^{10}+4 x^{9}-2 x^{8}-x^{6}-2 x^{5}-3 x^{4}-2 x+5$ \\
$x^{3}+6 x^{2}+8 x+5$ & $(x-1)^{2}\left(x^{2}+1\right)\left(x^{3}+1\right)$ & $x^{6}-x^{5}-3 x^{4}-3 x^{3}-2 x^{2}-x+5$ \\
$x^{3}+6 x^{2}+9 x+5$ & $\left(x^{2}+1\right)(x-1)^{2}$ & \\
\hline & &
\end{tabular}

\section{References}

[AG] S. Akiyama and N. Gjini, Connectedness of number theoretic tilings, Preprint.

[B] C. Bandt, Self-similar sets 5. Integer matrices and fractal tilings of $\mathbb{R}^{n}$, Proc. Amer. Math. Soc., 112 (1991), 549-562.

[BG] C. Bandt and G. Gelbrich, Classification of self-affine lattice tilings, J. London Math. Soc., 50 (1994), 581-593.

[BW] C. Bandt and Y. Wang, Disk-like self-affine tiles in $\mathbb{R}^{2}$, Discrete Comput. Geom., 26 (2001), 591-601.

[G1] I. Gargantini, The numerical stability of the Schur-Cohn criterion, SIAM J. Numer. Anal., 8 (1971), 24-29.

[G2] A. Garsia, Arithmetic properties of Bernoulli convolutions, Trans. Amer. Math. Soc., 102 (1962), 409-432.

[H] D. Hacon, N. Saldanha, and J. Veerman, Remarks on self-affine tilings, Experiment. Math., 3 (1994), 317-327.

[KL] I. Kirat and K. S. Lau, On the connectedness of self-affine tiles, J. London Math. Soc., 62 (2000), 291-304.

[L] K. S. Leung, Private communication.

[LW1] J. C. Lagarias and Y. Wang, Integral self-affine tiles in $\mathbb{R}^{n}$, I. Standard and non-standard digit sets, J. London Math. Soc., 54 (1996), 161-179. 
[LW2] J. C. Lagarias and Y. Wang, Integral self-affine tiles in $\mathbb{R}^{n}$, II. Lattice tiling, J. Fourier Anal. Appl., 3 (1997), 1-20.

[O] A. M. Odlyzko, Non-negative digit sets in positional number systems, Proc. London Math. Soc., 37 (1978), 213-229.

[T] B. Tan, Private communication.

Received March 20, 2002, and in revised form April 17, 2003. Online publication December 19, 2003. 